\title{
Scaling of Fluctuations in Internet - the State States Perspective
}

\author{
M. Bigus-Kwiatkowska ${ }^{a}$, A. FronczAK $^{a}, \mathrm{P}_{\text {. FronczaK }}^{a, *}$, \\ G. SiUdem ${ }^{a}$ AND M. THELWALL ${ }^{b}$
}

\author{
${ }^{a}$ Faculty of Physics, Warsaw University of Technology, \\ Koszykowa 75, PL-00662 Warsaw, Poland \\ ${ }^{b}$ Statistical Cybermetrics Research Group, School of Mathematics and Computing, \\ University of Wolverhampton, Wulfruna Street, Wolverhampton WV1 1SB, United Kingdom
}

Doi: $10.12693 /$ APhysPolA.138.14

*e-mail: piotr.fronczak@pw.edu.pl

\begin{abstract}
Taylor's law of fluctuation scaling is a power-law relationship between a variance and a mean. It has been observed in a wide range of disciplines, ranging from population dynamics through the Internet to the stock market. To explain origins of the law, a number of theoretical approaches have been proposed. Here, we discuss applicability of one of them, the maximum entropy-based formalism, to non-equilibrium stationary states. In addition to analyzing several real time series, we show that a simple model of transport of packets in communication networks also exhibits Taylor's law and could serve as a good testbed for verification of the formalism. We draw attention of the complex system community to the Bell polynomials that turn out to be very well suited for studying systems that exhibit Taylor's law.
\end{abstract}

topics: Taylor law, scaling fluctuation, Bell polynomials, density of states

\section{Introduction}

For the first time, Taylor's fluctuation scaling was observed in ecology [1] as the species-specific powerlaw relationship between the temporal or spatial variance $\sigma^{2}$ of populations, and their mean abundances $\langle N\rangle$ :

$$
\left\langle N^{2}\right\rangle-\langle N\rangle^{2}=a\langle N\rangle^{b}
$$

where the characteristic exponent $b$ describes effects of heterogeneity in spatial or temporal patterns of the frequency distribution. The exponent was of particular interest as it served as an index of aggregation: (i) $b \simeq 0$ implies a nearly regular distribution, (ii) $b=1$ suggests a random (Poisson) distribution, and (iii) $b>1$ indicates higher degrees of aggregation. In the vast majority of observations, the value of $b$ is in the range between 1 and 2 .

Apart of ecology, Taylor's law also manifests with other seemingly disparate processes such as the transmission of infectious diseases [2], human sexual behavior [3], cancer metastases [4], blood flow heterogeneity [5], as well as traffic on highways [6] and crime statistics [7]. Recently, when a huge amount of data has become available in Internet, Taylor's fluctuation scaling has aroused interest among physicists and computer scientists [8, 9]. Prevalence of Taylor's law was demonstrated in a number of virtual systems, including the Internet and the world wide web [9]. Simultaneously with the observations of Taylor's law a vast amount of models has been developed ranging from animal behavior [10], a random walk model [11] or a stochastic birth, death, immigration and emigration model [12] - each specific to one individual situation. But given such a broad applicability, one might ask whether some general principle might be at the basis of all these processes. Is there a mechanism able to explain the manifestations of Taylor's law without going into details of a particular system?

One possible mathematical explanation for the origin of Taylor's law is given by a class of Tweedie exponential dispersion models [13]. In analogy to the central limit theorem that governs the convergence behavior of certain types of random data, the Tweedie convergence theorem implies that Taylor's law results from a general mathematical convergence effect observed in Tweedie models.

In [6], Taylor's law has been derived from the maximal entropy principle (MEP) which is also a basis of equilibrium and non-equilibrium statistical physics - the approach that seems to be much more attractive and intuitive for physicists. In [6], the relation of the aggregation phenomena to the power law exponent $b$ has been explained through the well-defined density of states (DOS) function that encodes the distribution of system elements into clusters. The approach has been verified in a number of ecological, technological and economical systems.

The aim of this paper is twofold. Firstly, we demonstrate that the MEP-based formalism (naturally arising in the study of fluctuations in equilibrium ensembles) works well also in 
non-equilibrium stationary states and can explain temporal fluctuations. Secondly, since the available real data are limited and noisy, we adopt a simple model of transport of packets in communication networks that exhibits Taylor's temporal fluctuation scaling and could serve as a good testbed for the verification of the theoretical MEP-based model. Since the model exhibits continuous phase transition, we are also able to investigate aggregation phenomena and the way they are reflected in the discussed formalism in the vicinity of the critical point.

The outline of the paper is as follows. First, we describe real datasets which we use in our further analysis. Then, we introduce the model of packets traveling through the network. Next, we present theoretical analysis of MEP-based formalism and verify its elements against the real data and numerical time series obtained from the model. Finally, we shortly conclude the obtained results.

\section{Data description}

We have analyzed three datasets related to three different human activities on the Internet.

- Web page visits. Dataset came from the visitor statistics system of the NI Direct website [14], the official government website for Northern Ireland citizens. It contains daily visits of web pages in the period from 1st January 2018 to 31st March 2018. The short period ensures data stationarity. From all web pages we discarded those with time series containing zeroes (probably for some reasons inactive in the course of the analyzed period). In total, we had 1516 time series, each consisting of 90 ticks (days of 1st Quarter 2018). Taylor's law for these time series is shown in Fig. 1a.

- Blogs. Data represent weekly numbers of RSS feeds from more than 14000 blogs from various platforms (mainly MSN Spaces) [15]. Each time series represents a single blog and contains 20 ticks spanning the period of 20 weeks. Once again, the period is sufficiently short to avoid the problem of nonstationarity (i.e., increasing trend of the use of blogs). To eliminate blogs which become inactive in the course of the period we select only such time series which do not contain zeroes (i.e., each week there is non zero number of posts). Finally we had 1413 series in the period from 28.09.2007 to $14.02 .2008,1177$ series in the period from 15.04.2008 to 1.09.2008, 1045 series in the period from 16.11.2008 to 4.04.2009 and 663 series in the period from 7.07.2009 to 23.11.2009. In total, we had 4298 time series for which we can calculate the mean and variance resulting in Taylor's law shown in Fig. 1b.

- Packets. The data are measurements made on each packet of information that passes through the University of Auckland Internet Gateway [15]. These measurements include a timestamp that represents the time at which the packet reached the network location, and the size of the packet,
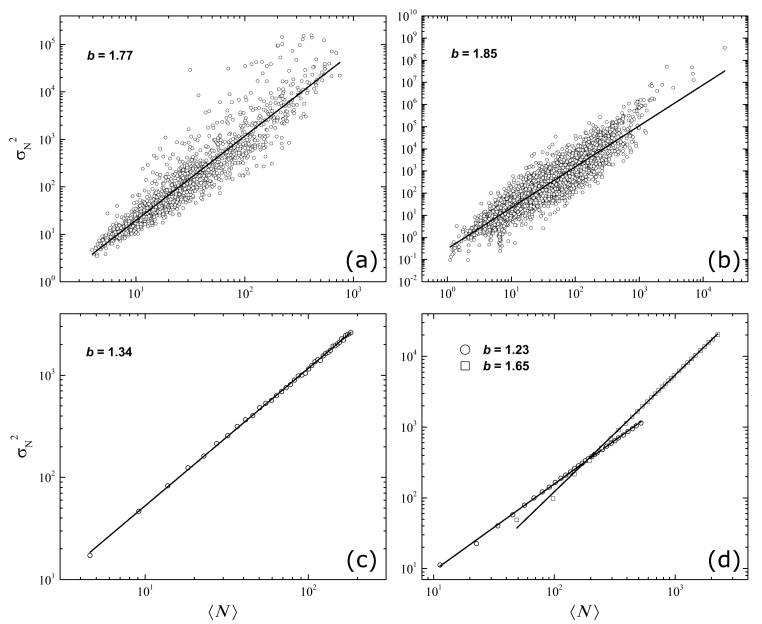

Fig. 1. Taylor's power law for scaling of fluctuation observed in: (a) web page visits, (b) blogs, (c) packets, and (d) the model of packet transport in the network of $N=500$ nodes and $m=1, k=20$ (open squares) and $m=2, k=60$ (open circles).

as a number of kilobytes. After data preprocessing, we obtained time series containing 20000 numbers spanning the period of $20 \mathrm{~s}(\mathrm{kB} / \mathrm{ms})$. The period is sufficiently short to avoid the problem of nonstationarity (daily variation in traffic load). Taylor's law for these data is shown in Fig. 1c. Please note, that since we had one time series only, we obtained points presented in Fig. 1c using the method of expanding bins [16] which will be described later.

\section{Model of packet transport in communication networks}

In the basic models frequently used to mimic transport phenomena in communication networked systems [17-19], all $M$ nodes in a network are equally considered as hosts and routers for generating and delivering packets. At each time step, there are $R$ packets generated in the system, with randomly chosen sources and destinations (here, for simplicity, we assume $R=1$ ). During the next time steps packets travel randomly (as a simple nonpreferential random walk) around the network, and look for their destination-nodes. Once a packet arrives at its target, it is removed from the system. If there are no other constrains, such as limited ability of nodes to distribute a large number of packets, the system evolves to a non-equilibrium stationary state. In such a state the number of packets $n_{i}(t)$ in a given node $i$ fluctuates around its mean value $\left\langle n_{i}\right\rangle$ with a variance $\sigma_{i}^{2}$. On the other hand, if nodes can deliver at most $C$ packets per time step towards their destinations, then a critical capacity $C_{\text {crit }}$ exists at which a continuous phase transition from free flow state to congested state occurs. In this jammed state, the number of packets accumulated in a node increases with time due to the limited delivering capacity. 
In this paper, we use the well-known BarabásiAlbert (BA) model [20] to generate the topology of the underlying network. In the BA model one starts from $m_{0}$ fully connected nodes, and new nodes, with $m$ edges each, are added sequentially to the existing nodes with the preference proportional to their degrees $k$. The growth process is stopped when the final network size is $M$. In our analysis, we need to extend the BA model a bit. Namely, we allow $m$ to be non-integer value. Such values of $m$ can be attained by adding a new node with $\lceil m\rceil$ edges (i.e., ceiling function, least integer greater than $m$ ) with probability $m+1-\lceil m\rceil$, and with $\lceil m\rceil-1$ edges otherwise.

Despite its simplicity, the BA model is a minimal model that reproduces the two important aspects observed in real networks: power-law function for the node degree distributions and small world effect understood as a fact that most pairs of nodes in real networks are connected by a relatively short path. As an additional advantage of this model, especially in the context of transport phenomena, one can recognize the fact that there is only one component in the network and no separated nodes exist (unlike other models that also reflect the first two aspects of real networks, e.g. configurational model [21] or exponential random graph model [22]).

Having the structure of underlying network established, we start to generate new packets. Let us assume for now, that the node capacity $C \rightarrow \infty$. The number of packets in the network $n(t)$ initially increases, but after some transient time $t_{\text {init }}$ it saturates and start to fluctuate around some mean value $\langle n\rangle$. Now, starting from $t_{\text {init }}$, we construct $M$ time series $\Lambda_{i, 1}$, where each $\Lambda_{i, 1}$ (the subscript 1 is used to ensure consistency with further labeling) represents the number of packets $n_{i}(t)$ in the node $i$ and $t_{\text {init }}<t<t_{\max }$. The length of each time series is then $L_{i}=t_{\max }-t_{\text {init }}$.

To analyze Taylor's law in these time series (as well as, in datasets coming from real measurements, see Fig. 1c) we use the method of expanding bins [26]. In this method, a time series $\Lambda_{i, 1}$ is divided into a set of non-overlapping bins of size $s$, where $1 \leq s \ll L_{i}$. Then, a new time series $\Lambda_{i, s}$ of length $L_{i} / s$ is constructed, in which each element is just a sum of packets in a respective bin. Now, one can calculate mean and variance for different $s$. In Fig. 1d, we present fluctuation scaling obtained by this method for two different nodes with degree $k=20$ and $k=60$ in two different networks with $M=500$ and $m=1$ and $m=2$, respectively.

It is worth to discuss for a moment the differences in power-law exponent $b$ that are visible in Fig. 1d. To understand these differences we have calculated $b$ for all the nodes in the network and verify its dependency on the node degree $k_{i}$. We have also performed such an analysis for networks that differ in the parameter $m$. The results of the both analyses are presented in Fig. 2.

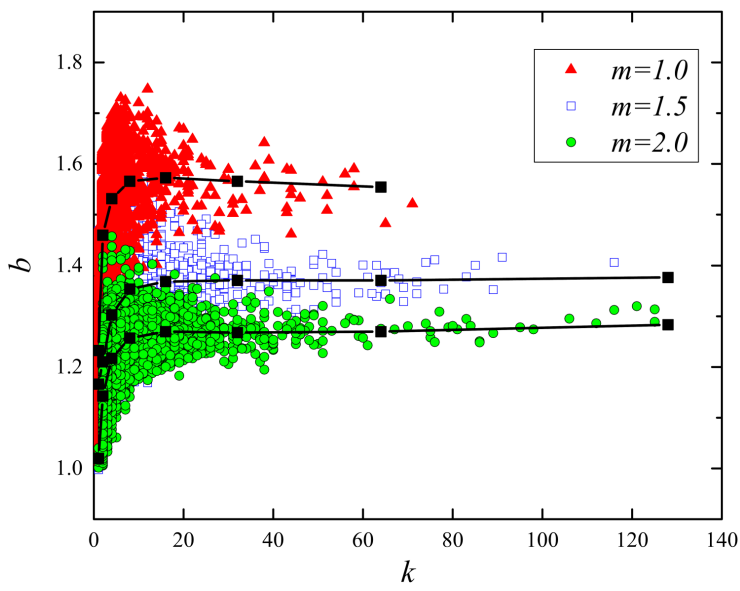

Fig. 2. Scaling exponent $b$ in Taylor's power law for different node degrees $k$ in three different networks with $M=500$ and $m=1$ (red triangles), $m=1.5$ (blue squares), and $m=2$ (green circles). Black lines and points show $b$-value averaged over similar node degree and logarithmically binned.

The most striking observation is that the exponent $b$ is negatively correlated with the network density. The largest values of $b$ have been obtained for tree-like networks with $m=1$. For $m \gg 1, b \rightarrow 1$, and the load fluctuations become Poissonian. Moreover, the node degree seems to play a secondary role in a variability of $b$, which, averaged over nodes with the same degree, is constant for $k>10$ see black lines in Fig. 2). At first glance, the dispersion of $b$ around its mean value for a given node degree should be related to the differences in local neighborhood of a node. However, we have studied $b$-dependence on the average nearest neighbor node degree for all the nodes with $k=1$ and did not find any clear relationship. Thus, other non-local properties of nodes seem to be responsible for this dispersion (like betweenness centrality and other flow related characteristics).

To summarize, the presented model of packet transport exhibits the desired power law of fluctuations with the exponent in a range $1<b<1.75$, what complies with the ubiquity of empirical Taylor's power-law slopes in the interval $1<b<2$. Moreover, since the lengths $L_{i}$ of time series are almost unlimited, uncertainty of the further analysis related to noisy and limited experimental data can be effectively reduced.

\section{Main analysis}

In this section, we verify the elements of the MEP-based formalism against the real and simulated data. To make this section readable we must reproduce here (in shortened version) some of the results of the paper [6], where the formalism was presented for the first time.

General solution for the maximum entropy distribution $\mathcal{P}(\Omega ; \mu)$ constrained to yield the average 
value of the parameter $N$ (the number of counts, e.g. packets or web page visits, over time) is given by

$$
\mathcal{P}(\Omega ; \mu)=\frac{\mathrm{e}^{-\mu N(\Omega)}}{\mathrm{e}^{F(\mu)}}
$$

where $\mu$ stands for the external field coupled to $N$ that imposes a given value of $\langle N\rangle$, and $\mathrm{e}^{F(\mu)}$ is normalization factor of the distribution. In statistical physics, $F(\mu)$ represents the so-called free energy of the considered system that encodes properties of the system in equilibrium. In what follows, we also call it free energy. Greek letter $\Omega$ in (2) refers to the so-called microstate of the considered system, whereas researchers studying real-world systems are usually interested in macrostates and the corresponding macroscopic quantities. Macroscopic states are characterized by the frequency distribution $P(N ; \mu)$ and the two introduced distributions are related to each other by:

$$
P(N ; \mu)=g(N) \mathcal{P}(\Omega ; \mu),
$$

where $g(N)$ is the announced density of state function (DOS), which gives the number of microstates having the same value of the macroscopic parameter $N$. Given the above equations we assume that the system can exchange its components with a large reservoir in equilibrium. An answer to the question: "What is reservoir and external field $\mu$ in social systems?" is not simple. In the case of web page visits we may say that there is a large (constant in a considered time span) population of Internet users who spent their time visiting different web pages. A field $\mu$ can represent ability of a given webpage to attract the users' attention.

To make us sure that the above assumptions, i.e., (2) and (3), are a good starting point for further analysis we could perform a simple test (no. 1) based on the observation that the quotient of two frequency distributions corresponding to different average values of $\langle N\rangle$ is an exponential function of $N$, namely

$$
\frac{P\left(N ; \mu_{1}\right)}{P\left(N ; \mu_{2}\right)} \propto \mathrm{e}^{\left(\mu_{2}-\mu_{1}\right) N} .
$$

If we select two time series corresponding to different parameters $\mu_{1}$ and $\mu_{2}$ (what imposes two different means $\left\langle N_{1}\right\rangle$ and $\left\langle N_{2}\right\rangle$ ), then calculate respective frequency distributions, and take their quotient, we should get a straight line in the semi log plot of the quotient as a function of $N$. Figure 3 presents the results of this simple test with reference to both real world datasets, as well as to the packet transport model. As one can see, the results agree very well with theoretical expectations.

In the following, we will try to find explicit formula for frequency distribution $P(N ; \mu)$. To derive this formula from (2) and (3) one has to know the three following terms: the value of external field $\mu$ corresponding to the given mean $\langle N\rangle$, functional dependence $F(\mu)$ of free energy on $\mu$, and the DOS function $g(N)$.
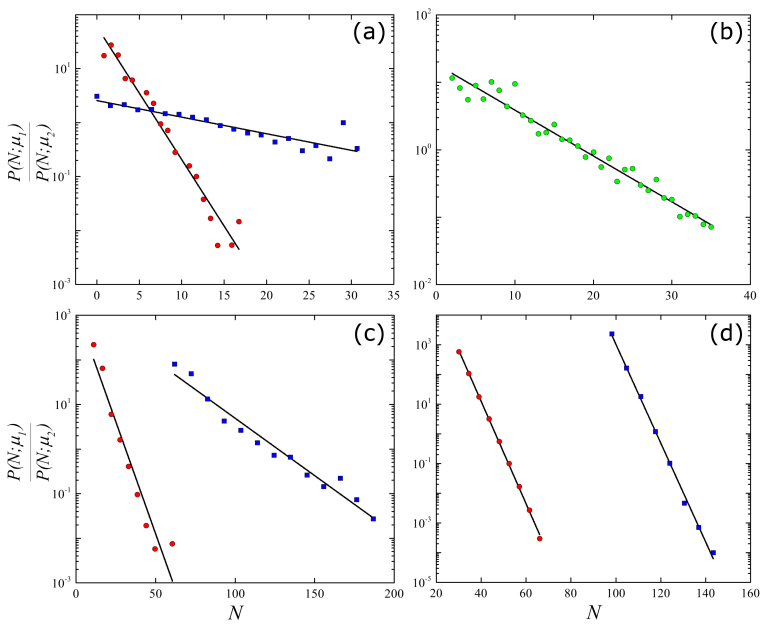

Fig. 3. Results of the test no. 1: (a) web pages - parameters $\mu_{1}$ and $\mu_{2}$ correspond to the means $\left\langle N_{1}\right\rangle=5.234$ and $\left\langle N_{2}\right\rangle=18.67$ (red circles), $\left\langle N_{1}\right\rangle=10.10$ and $\left\langle N_{2}\right\rangle=11.81$ (blue squares), (b) blogs $-\left\langle N_{1}\right\rangle=13.8$ and $\left\langle N_{2}\right\rangle=25.0$ (green circles), (c) packets $-\left\langle N_{1}\right\rangle=9.14$ and $\left\langle N_{2}\right\rangle=59.39$ (red circles), $\left\langle N_{1}\right\rangle=82.23$ and $\left\langle N_{2}\right\rangle=164.5$ (blue squares), (d) model of packet transport $-\left\langle N_{1}\right\rangle=$ 33.9 and $\left\langle N_{2}\right\rangle=56.51$ (red circles), $\left\langle N_{1}\right\rangle=88.96$ and $\left\langle N_{2}\right\rangle=148.3$ (blue squares). The straight lines are the best fitting by the least squares criterion.

Two first elements can be easily calculated exploiting another fundamental statistical physics formula, i.e., fluctuation-dissipation relation. It is defined by

$$
\left\langle N^{2}\right\rangle-\langle N\rangle^{2}=-\frac{\partial\langle N\rangle}{\partial \mu}=\frac{\partial^{2} F(\mu)}{\partial \mu^{2}},
$$

which states that fluctuations of the parameter $N$ are proportional to susceptibility of the parameter to its conjugate field $\mu$. Comparing right-hand sides of (5) and (1), one obtains differential equation for $\langle N\rangle$, i.e., $-\partial\langle N\rangle / \partial \mu=a\langle N\rangle^{b}$. Solving this equation, with the reasonable assumption of nonnegative variance, one obtains

$$
\langle N\rangle= \begin{cases}X \mathrm{e}^{-a \mu} & \text { for } b=1 \\ {[(b-1) a \mu+X]^{1 /(1-b)}} & \text { for } b>1\end{cases}
$$

Here, $X$ represents integration constant, which could be later easily eliminated through the probability normalization. Next, having (6) and again exploiting (5) by solving $\partial F / \partial \mu=-\langle N\rangle$, one also finds the formula for the free energy $F(\mu)$ :

$$
F(\mu)= \begin{cases}\frac{1}{a(2-b)}\langle N\rangle^{(2-b)}+Y & \text { for } b \geq 1 \\ \frac{1}{a} \ln \langle N\rangle+Y & \text { for } b=2 .\end{cases}
$$

In the right hand side of (7), the parameter $\mu$ is expressed implicitly through $\langle N\rangle$. From the fact, that in the empty system (i.e., when $\langle N\rangle=0$ ) there is no energy at all, the integration constant $Y$ can be set to be equal to zero.

Before go further, to be sure that we are correct about our assumptions (e.g. about applicability of fluctuation-dissipation theorem to so unphysical 


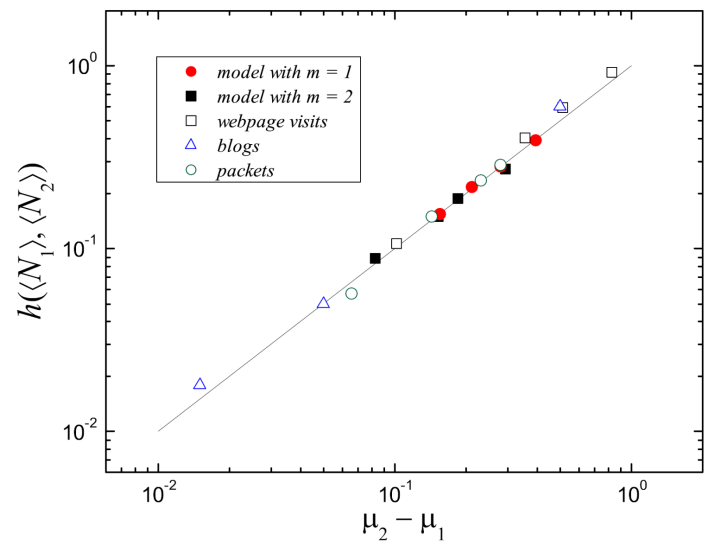

Fig. 4. Results of the test no. 2. The indicated line represents the $y=x$ diagonal line.

systems as ours) and derivations so far, we can perform the test no. 2. Transforming the expression for $\langle N\rangle$, one can show that the experimental data should satisfy the identity

$$
\mu_{2}-\mu_{1}=\frac{\left\langle N_{2}\right\rangle^{1-b}-\left\langle N_{1}\right\rangle^{1-b}}{a(1-b)}=h\left(\left\langle N_{1}\right\rangle,\left\langle N_{2}\right\rangle\right) .
$$

The value of the left hand side of (8) can be easily extracted from the slope of the linear fit, which is shown in Fig. 3. Taking many different pairs of frequency distributions $P\left(N ; \mu_{1}\right)$ and $P\left(N ; \mu_{2}\right)$ one can obtain a group of points which all should lie on the straight line characterized by (8). The results of the test are shown in Fig. 4. Once again, the agreement with theoretical expectations is convincing.

The above derivations reveal the first two elements needed to find an explicit expression for frequency distribution $P(N ; \mu)$. These elements are the external field $\mu$ that corresponds to the mean $\langle N\rangle$, and the functional form of the free energy, given by (6) and (7), respectively.

In fact, the third lacking element — DOS function — is also accessible. In [6], a general combinatorial formula for DOS function was presented:

$$
g(N) \simeq \frac{\mathrm{e}^{-N}}{N !} B_{N}\left(f_{1}, f_{2}, \ldots, f_{N}\right),
$$

where $B_{N}\left(f_{1}, f_{2}, \ldots, f_{N}\right)$ is the $N^{\text {th }}$ complete Bell polynomial whereas $f_{n}$ represents the coefficient of the $n^{\text {th }}$ term in the MacLaurin expansion of the free energy. Since the expression for the free energy (7) is known, we can easily calculate $f_{n}$, as the $n^{\text {th }}$ derivative of $F(\mu)$ at $\mu=0$.

Now, before proceeding, we have to stop and explain more deeply the meaning of the complete Bell polynomial, since for most of physicists it is unknown combinatorial creature. In general, the $N^{\text {th }}$ complete Bell polynomial, $B_{N}\left(f_{1}, f_{2}, \ldots, f_{N}\right)$, describes the number of disjoint partitions of a set of size $N$ into an arbitrary number of subsets [23]. The parameters $f_{i}$, with $i=1,2, \ldots, N$, apply to subsets of size $i$ and play an important role in a description of the partitions.

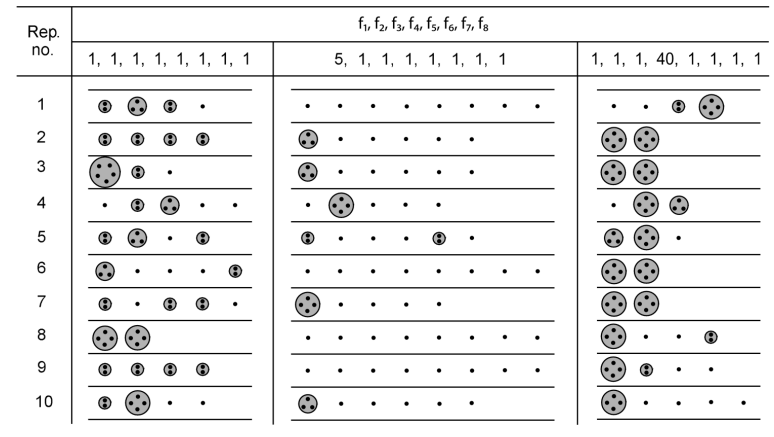

Fig. 5. Three examples of partitions of a set of $N=8$ elements. Detailed description is given in the text.

For example, if the all parameters $f_{1}, f_{2}, \ldots, f_{N}$ have the same value, then there is no preference for the size of subsets. The resulting partitions correspond to a random distribution of elements, the distribution of subset sizes is uniform, and the frequency distribution describing that system, $P(N)$, is Poissonian. On the other hand, in the extreme case of $f_{i} \gg f_{j}$ for all $j \neq i$, the Bell polynomial gives the number of such partitions in which there is a strong preference for subsets of size $i$.

To be better understood we present in Fig. 5, three examples of partitions of a set of $N=8$ elements. In each example there is a different preference for the subset size. Since the number of ways of partitions of even small set can be enormous, we select from the whole ensemble of partitions ten randomly chosen representatives. In Fig. 5a, all $f_{1}, f_{2}, \ldots, f_{N}$ have the same value and each partition is composed of completely random clusters (with the constrain that their total mass is $N$ ). Examples shown in Figs. 5b and 5c prefer subsets of size 1 and 4, respectively.

Although the combinatorial expression for DOS function (9) is general and can be applied to any statistical system, in the case of systems in which Taylor's law manifests itself, it is very meaningful. It's straight interpretation relates directly DOS function with the aggregation phenomena observed in real world system.

Now, we can perform the final test (no. 3) as we have already derived all necessary expressions for evaluating the frequency distribution $P(N ; \mu)$. The only problem is that the real world data frequency distribution has to be estimated from very few measurements (for example, we have 20 and 90 values in time series of blogs and page visits respectively), which is completely insufficient. To increase the amount of data from which a distribution is made, we have assumed that the neighboring points in the mean-variance graph result from similar environmental conditions (i.e., from similar values of the parameter $\mu$ ). In this way, a single smooth distribution possessing the given values of $\langle N\rangle$ and $\sigma_{N}^{2}$ has been prepared as a simple sum of all the component distributions corresponding to 

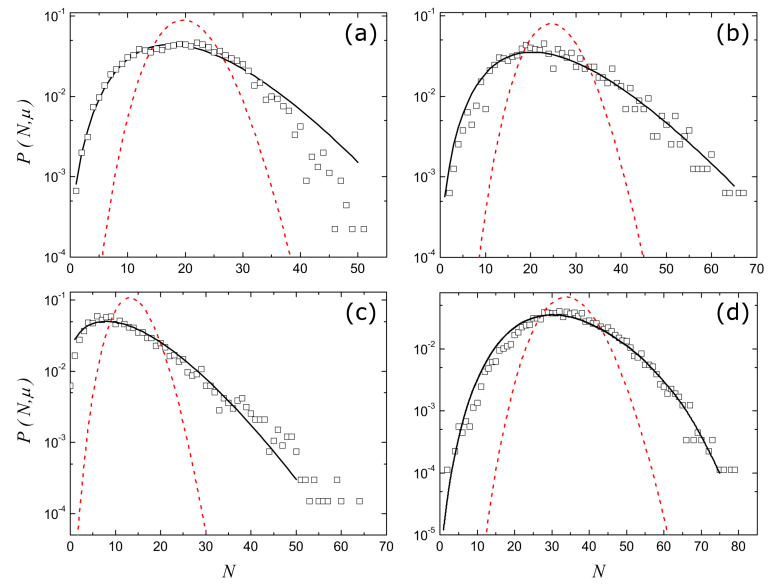

Fig. 6. Theoretical (solid black line) and experimental (open squares) frequency distributions $P(N ; \mu)$ as well as the Poisson distribution with the same mean (dashed red line) for (a) web page visits with $\langle N\rangle=20.1$, (b) blogs with $\langle N\rangle=24.2$, (c) packets with $\langle N\rangle=13.7$, and (d) model of packet transport with $M=500, m=1, k=1$ and $\langle N\rangle=34$.

single points $\left[\left\langle N_{i}\right\rangle, \sigma_{N_{i}}^{2}\right]$ in the mean-variance graph, and meeting the following conditions:

$$
\begin{aligned}
& \frac{\left\langle N_{i}\right\rangle}{d}<\langle N\rangle\left\langle d\left\langle N_{i}\right\rangle\right. \\
& \frac{\sigma_{N_{i}}^{2}}{d}<\sigma_{N}^{2}<d \sigma_{N_{i}}^{2} .
\end{aligned}
$$

Parameter $d$ describes a linear size of the square in the log-log plot in the mean-variance graph with the central point of the square $\left[\langle N\rangle, \sigma_{N}^{2}\right]$ placed in the solid line corresponding to the empirical Taylors law. If $d$ is chosen to be too small $(d \rightarrow 1)$, then the square shrinks to the single point, what leads to poor data sampling and noisy distribution. On the other hand, when the parameter $d$ is taken too large, the resulting distribution, $P(N ; \mu)$, is made of distributions that characterize rather different environmental conditions. Although the obtained distribution is smooth, it is not very reliable. This reliability can be verified observing the shape of the quotients $P\left(N ; \mu_{1}\right) / P\left(N ; \mu_{2}\right)$ in Fig. 2. For sufficiently small values of $d$ the shape will be stable. Taking above into consideration we have chosen $d=1.2$. The above procedure allows us to increase the number of points to several hundreds or even more. Of course, the above procedure does not apply to data generated using the model of packet transport, where we are not limited by short time series.

Now, we can proceed with the third test. Its results are shown in semi-log scale in Fig. 6. The figure consists of four parts - each of them presenting different case. Each panel illustrates by comparison the theoretical and experimental frequency distributions $P(N ; \mu)$, as well as the Poisson distribution with the same mean. Taking into consideration
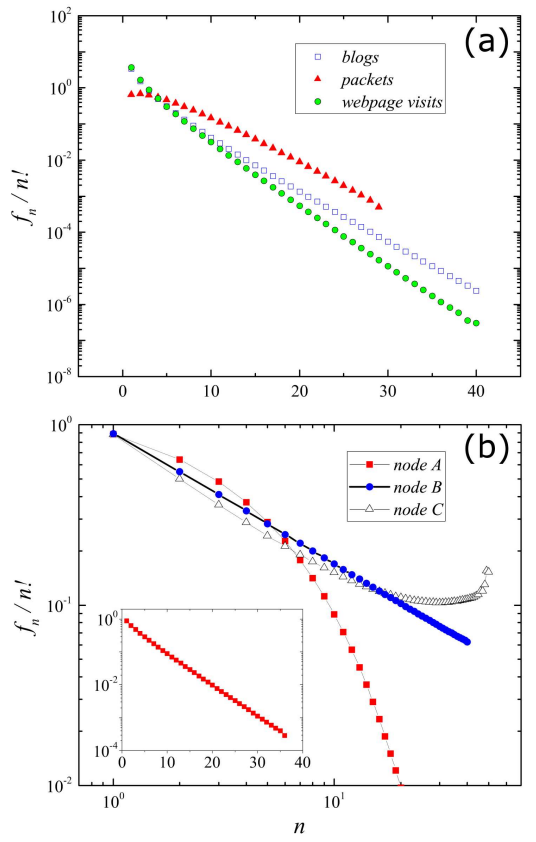

Fig. 7. The coefficients $f_{n}$ in the series expansion of the free energy (9) for: (a) webpage visits (green circles), blogs (blue squares) and packets (red triangles), b) three different nodes in the model of packet transport (parameters used are: $N=50, m=3$, $C=1, R=3$ ) with degrees $k=6$ (node $\mathrm{A}$ ), $k=10$ (node $\mathrm{B}$ ), and $k=11$ (node $\mathrm{C}$ ).

the noisy environment in which the data have been gathered, our theory seems to be in excellent agreement with the data.

Finally, let us look closer at the coefficients $f_{n}$ in the series expansion of $F(\mu)$. If the free energy (9) is divided by $n$ ! (to remove distinguishability of events), then $f_{n}$ 's may be interpreted as thermodynamic preferences for clusters of size $n=1,2, \ldots$. Since we consider time series with temporal fluctuations, one should remember that these clusters are not spanned in space but they represent time intervals with high intensity of human activity separated by the moments when none or small Internet activity is present [24]. In Fig. 7a, these preferences are presented for all real data sets in our collection. They all behave in the same manner, namely they are exponentially decreasing functions of $n$.

Such a behavior resembles the one observed in the two-dimensional Ising model, where the coefficients in the low temperature series expansion of the free energy have a similar asymptotic behavior [25]. It was shown that the phase transition in the Ising model can be explained through equivalence between the model and the perfect gas of energy clusters model, in which the passage through the critical point is related to the complete change in the thermodynamic preferences on the size of clusters. Below the critical temperature, i.e., in the ferromagnetic state, smaller energy clusters are characterized by higher preferences, while, above the critical temperature, the 
preferences monotonically increase as a function of $n$. Phase transition occurs, when the preferences do not depend on clusters' size.

Although the real datasets do not allow us to observe such a transition, in the model of packet transport the phase transition from free flow state to congested state is possible when the capacity $C$ of nodes is reduced [26]. Figure $7 \mathrm{~b}$ presents the situation, when the system is in the critical state $C \sim C_{\text {crit }}$. Please note, that in heterogeneous networks nodes of different degrees become congested for different values of $C$ [27]. It also means that the system still possesses partial capacity for forwarding packets in this phase, although as a whole, it enters the jammed state even if one node is congested. In Fig. 7b, coefficients $f_{n}$ for three different nodes are presented. The node A of degree $k=6$ is a node in which free flow of packets is still possible. The cluster preferences decay exponentially in the same manner as in real data (see inset in Fig. 7b). The flow in the node $\mathrm{B}(k=10)$ is critical. Cluster preferences follow a power law function. The node $\mathrm{C}(k=11)$ is jammed. Although it does not fulfill the condition of stationarity (the number of packets in the node slowly increases), one can observe that the preference for large clusters becomes meaningful. All nodes with the larger degrees are congested, and the above analysis could not be performed.

\section{Concluding remarks}

In the paper, we have demonstrated application of MEP-based formalism of Taylor's fluctuation scaling to temporal data. In addition to real data, we also used data from the packet transport model in complex network. Their advantage over real data comes from the fact that these time series obtained from the model do not have length limitations and, because not influenced by external factors, their statistical properties such as mean, variance, autocorrelation, etc., are all constant over time. The ability of the model to capture phase transition between free flow and congested state allowed us to discuss the influence of the coefficients $f_{n}$ in the series expansion of the free energy of the system on the clustering of time series. Finally, we believe that the Bell polynomials approach, though almost unnoticed by physicists who deal with complex systems, is well suited to the study of such clustered systems. We hope that this work will bring this formalism a little closer to the complex system society.

\section{Acknowledgments}

This work has been supported by the $\mathrm{Na}$ tional Science Centre of Poland (Narodowe Centrum Nauki, NCN) under grant no. 2012/05/E/ST2/02300. M.B. and G.S. performed numerical simulations, A.F. designed and coordinated the project, P.F. wrote manuscript, M.T. collected data.

\section{References}

[1] L.R. Taylor, Nature 189, 732 (1961).

[2] C.J. Rhodes, R.M. Anderson, Nature 381 , 600 (1996).

[3] R.M. Andersen, R.M. May, Nature 333 , 514 (1988).

[4] Wayne S. Kendal, J. Theor. Biol. 217, 20 (2002).

[5] Wayne S. Kendal, Proc. Natl. Acad. Sci. (PNAS) 98, 837 (2001).

[6] A. Fronczak, P. Fronczak, Phys. Rev. E 81, 066112 (2010).

[7] Q.S. Hanley, S. Khatun, A. Yosef, R.-M. Dyer, PLOS ONE 9, e109004 (2014).

[8] Z. Eisler, I. Bartos, J. Kertész, Adv. Phys. 57, 89 (2008).

[9] M. Argollo de Menezes, A.-L. Barabási, Phys. Rev. Lett. 92, 028701 (2004).

[10] R.A.J. Taylor, L.R. Taylor, Nature 265 , 415 (1977).

[11] I. Hanski, Oikos 34, 293 (1980).

[12] R.M. Anderson, D.M. Gordon, M.J. Crawley, M.P. Hassell, Nature 296, 245 (1982).

[13] W.S. Kendal, Ecol. Complex. 1, 19 (2004).

[14] NI Direct website data.

[15] P. Murrel, The Network Packets Data Set.

[16] Wen-Xu Wang, Bing-Hong Wang, ChuanYang Yin, Yan-Bo Xie, Tao Zhou, Phys. Rev. E 73, 026111 (2006).

[17] R. Guimerá, A. Arenas, A. Diaz-Guilera, F. Giralt, Phys. Rev. E 66, 026704 (2002).

[18] Liang Zhao, Ying-Cheng Lai, Kwangho Park, Nong Ye, Phys. Rev. E 71, 026125 (2995).

[19] B. Tadić, S. Thurner, G.J. Rodgers, Phys. Rev. E 69, 036102 (2004).

[20] R. Albert, A.-L. Barabási, Rev. Mod. Phys. 74, 47 (2002).

[21] M. Molloy, B. Reed, Comb. Probab. Comput. 7.3, 295 (1998).

[22] Juyong Park, M.E.J. Newman, Phys. Rev. E 70, 066117 (2004).

[23] L. Comtet, Advanced Combinatorics: the Art of Finite and Infinite Expansions, Rev. Ver., Springer, Dordrecht 1974.

[24] A.-L. Barabási, Nature 435, 207 (2005).

[25] G. Siudem, A. Fronczak, P. Fronczak, Sci. Rep. 6, 33523 (2016).

[26] Liang Wu, Xin Yan, Jiang Du, Phys. Rev. E 90, 062808 (2014).

[27] P. Fronczak, Eur. Phys. J. B 85.10, 351 (2012). 